\title{
Minimal Change Disease as Initial Presentation of ALK-Positive Anaplastic Large-Cell Lymphoma in a Pediatric Patient
}

\author{
Karla Diaz ${ }^{\mathrm{a}}$ William Slayton $^{\mathrm{b}}$ Nirupama Guptac \\ aDivision of General Pediatrics, University of Florida, Gainesville, FL, USA; ${ }^{b}$ Division of \\ Hematology and Oncology, Department of Pediatrics, University of Florida, \\ Gainesville, FL, USA; 'Division of Nephrology, Department of Pediatrics, University of \\ Florida, Gainesville, FL, USA
}

\section{Keywords}

Immunology · Pediatric patient · Anaplastic large-cell lymphoma · Hemophagocytic lymphohistiocytosis · Nephrotic syndrome

\section{Abstract}

The association between nephrotic syndrome (NS), hemophagocytic lymphohistiocytosis $(\mathrm{HLH})$, and certain paraneoplastic syndromes has been documented in the literature. However, nephrotic changes as part of paraneoplastic syndromes are rare in lymphoid malignancies, particularly in non-Hodgkin lymphoma. We report the sudden onset of acute renal failure and NS in a 14-year-old male who initially presented with $\mathrm{HLH}$ and was subsequently diagnosed with ALK-positive anaplastic large-cell lymphoma (ALCL). The treatment of ALCL reversed both the HLH and NS findings. This case demonstrates the importance of considering lymphoma in pediatric patients presenting with NS and $\mathrm{HLH}$. 


\section{Case Reports in Oncology}

\section{Introduction}

Nephrotic syndrome (NS) is defined by proteinuria, hypoalbuminemia, and edema. Both hemophagocytic lymphohistiocytosis (HLH) and lymphoma via paraneoplastic syndrome can cause secondary NS. However, NS as part of a paraneoplastic syndrome is rare in lymphoid malignancies. We present a unique pediatric case of NS with HLH that was secondary to anaplastic large-cell lymphoma (ALCL).

\section{Case Report}

A 14-year-old male with a history of autism presented with multi-organ dysfunction and daily fevers of unknown etiology for a few days. He had upper respiratory infection symptoms and fatigue a few days prior to presentation at an outside hospital. His initial labs (Table 1) were significant for acute renal failure, hypoalbuminemia, leukopenia, and elevated liver enzymes, creatine kinase levels, and inflammatory markers. He was found to have significant proteinuria, with a rapid weight gain above his baseline. Given his acute onset of renal failure, a renal biopsy was performed at the outside hospital and hemodialysis initiated. The renal biopsy showed acute tubular dilation and interstitial damage with sparing of the glomeruli (Fig. 1).

Due to concern for autoimmune disease, he was transferred to our hospital for evaluation by the pediatric rheumatology, infectious disease, and nephrology units. A repeat renal biopsy at our facility confirmed the prior findings but with increased severity of podocyte damage. A diagnosis of minimal change NS was made. He required $50 \mathrm{~g}$ of $25 \%$ albumin $(1 \mathrm{~g} / \mathrm{kg})$ about twice to 3 times daily and prednisone $(2 \mathrm{mg} / \mathrm{kg}$, max. $60 \mathrm{mg})$ for NS therapy. He also underwent a liver and muscle biopsy due to elevated transaminases, coagulopathy, and myositis. The liver biopsy showed a few apoptotic bodies with regenerative changes, mild iron deposition, no cytoplasmic inclusions, and no fibrosis. The muscle biopsy showed nonspecific findings of myofiber necrosis and phagocytosis. An extensive rheumatologic workup was negative for C-ANCA, P-ANCA, EJ, histone antibody, KU, MI-2 autoantibody, P155/140 antibody, SSA 60 IgG antibody, SS-A antibody IgG, PL7, PL-12, Jo-1 antibody, OJ, PM-SCL antibody, SRP, U2 SN RNP, ANA, and rheumatoid factor. An infectious workup was negative for adenovirus, CMV, EBV, HHV6, HSV, parvovirus B19, enterovirus (CSF), and Rickettsia rickettsii-Rocky Mountain spotted fever. Blood, urine, and blood fungal cultures were also negative. Respiratory viral panel was positive for both rhinovirus and enterovirus.

Seven days after admission, a workup for anemia of chronic kidney disease raised suspicion for HLH (Table 2). After meeting 7 out of 8 criteria for HLH (fever, elevated ferritin $[14,742 \mathrm{ng} / \mathrm{mL}]$, splenomegaly, pancytopenia, hypertriglyceridemia, hemophagocytosis on bone marrow biopsy, and elevated soluble interleukin-2 receptor), treatment for HLH and NS was continued with high-dose steroids. However, despite improvement in HLH biomarkers and resolution of fever, severe NS persisted.

Twenty days after admission, daily fevers recurred, and a repeat infectious workup was negative for the earlier studies and the following: HIV, hepatitis A, B, and C, nonreactive RPR, Bartonella quintana, Ehrlichia chaffeensis, Mycoplasma, Borrelia burgdorferi, Babesia, cryptococcal antigen, and Aspergillus. Multiple blood and urine cultures tests were negative as well. 


\section{Case Reports in Oncology}

Case Rep Oncol 2017;10:964-972

DOI: $10.1159 / 00048185$

(c) 2017 The Author(s). Published by S. Karger AG, Basel www.karger.com/cro

Diaz et al.: Minimal Change Disease as Initial Presentation of ALK-Positive Anaplastic Large-Cell Lymphoma in a Pediatric Patient

A CT scan of the chest, abdomen, and pelvis showed hepatomegaly, ascites, and no discrete mass or drainable abscess. Pediatric rheumatology recommended anakinra, an interleukin-1 receptor antagonist, for possible juvenile idiopathic arthritis (JIA) being masked by steroids. However, anakinra was discontinued after 1 week after the fevers continued to persist. The infectious disease unit then recommended a gallium scan for an occult abscess evaluation, which showed intense uptake along the left iliac-psoas region (Fig. 2a). An MRI scan revealed extensive left retroperitoneal and left pelvic lymphadenopathy (Fig. 2b), which was missed on the initial CT scan due to low contrast use in the setting of renal failure. Subsequently, a femoral lymph node biopsy confirmed the diagnosis of ALK-positive ALCL.

After 1 month of chemotherapy, the patient slowly improved with resolution of fevers. NS completely resolved without requiring further albumin infusion after 3 months of chemotherapy. After 1 year of diagnosis, he had recurrence of ALCL, without features of HLH or NS. He was treated with crizotinib, and he is currently undergoing a reduced-intensity bone marrow transplant using his brother as a donor.

\section{Discussion}

To our knowledge, this is the first report of secondary minimal change disease (MCD) concurrently with secondary HLH and primary ALCL. Our patient presented with a diagnostic dilemma of overlapping features of acute renal failure with NS and HLH secondary to ALCL. Due to the presence of acute renal failure, the initial low-contrast CT scan missed the primary diagnosis of malignancy, which affected his overall treatment course. At first, he underwent therapy for NS with steroids with minimal improvement, and this may have delayed the recognition of lymphoma. Later, anakinra was added for suspicion of JIA. However, his renal and HLH manifestations resolved only after the correct diagnosis of ALCL and after lymphoma-directed chemotherapy was initiated.

HLH is a life-threatening condition due to an abnormal activation of cytotoxic T and natural killer cells, which leads to unregulated macrophage and lymphocyte proliferation. HLH is often a primary disease due to genetic disorders in pediatric patients or a secondary disease, where malignancy, infection, and autoimmune disorders are the most common triggers [1]. The diagnosis of HLH requires 5 of $8 \mathrm{HLH}-2004$ criteria [2]. The diagnosis of lymphomaassociated HLH requires the same criteria in addition to the documented histological evidence of a lymphoma. HLH in the context of malignancy can occur either concomitantly with (or even prior to) overt malignant disease. The prevalence of malignancy in children with suspected HLH is $8.4 \%$, of which most cases are due to T-cell malignancy or non-Hodgkin lymphoma (NHL) [3].

ALCL is a rare type of T-cell lymphoma accounting for 3-8\% of all lymphomas and 10$15 \%$ of all childhood NHL [4]. Renal manifestations in HLH and NHL are rare and often include acute kidney injury and/or NS. Acute kidney injury is attributed to acute tubular necrosis, hypoperfusion, tumor lysis, or glomerulopathy [5]. NS in children is most commonly seen as MCD. Primary MCD is a result of podocyte injury secondary to a circulating permeability factor affecting the glomerular filtration barrier, whereas secondary MCD can be due to drugs, infection, malignancy, and rheumatologic disorders. 


\section{Case Reports in Oncology}

Lehmberg et al. [3] showed a high prevalence of HLH triggered by ALCL (57\%); however, this study did not report the clinical manifestations at the time of diagnosis. In a large retrospective study of 50 children, the authors also did not comment on renal involvement as a feature of ALCL, with or without HLH [4]. A literature review showed no renal involvement in 9 other pediatric patients with HLH-associated ALCL [4]. Da'as et al. [6] reported 66 patients with renal involvement amongst 700 patients with NHL. Only 4 out of 37 NHL cases from the world literature at that time presented with MCD. Kofman et al. [7] reported 19 adult patients with biopsy-proven MCD from 201 patients with NHL. NS preceded, occurred simultaneously with, or followed the diagnosis of NHL. The lymphoproliferative disorders associated with MCD in this cohort were the following: chronic lymphocytic lymphoma $(22 \%)$, mantel cell lymphoma, peripheral T-cell lymphoma, marginal zone lymphoma (28\%), and Waldenström macroglobulinemia (33\%).

The treatment for secondary HLH includes medications, such as high-dose corticosteroids, etoposide, cyclophosphamide, and methotrexate, aimed to target the hyperinflammatory state. Recently, some studies reported that patients with HLH have elevated serum interleukin-1 levels and may benefit from interleukin-1 antagonist therapy [8]. However, these reports involved patients with JIA that developed macrophage activation syndrome and patients with secondary HLH due to infections. In our patient, anakinra did not show an appreciable response.

In summary, we present the first comprehensive report of a pediatric patient with secondary MCD in the setting of secondary HLH and primary ALCL. This case highlights the importance of a diagnostic workup and making the appropriate diagnosis to tailor medical management and drug therapy. The renal manifestations and secondary HLH both resolved after the correct diagnosis of ALCL and after initiation of lymphoma-directed chemotherapy. For physicians, the important message is to evaluate for lymphoma in a young adult presenting with HLH due to the strong association between HLH and malignancies.

\section{Statement of Ethics}

No animal or human experiments were conducted in this study. The authors have no ethical conflicts to disclose.

\section{Disclosure Statement}

The authors have declared that no conflict of interest exists.

\section{References}

-1 Filippone EJ, Farber JL: Hemophagocytic lymphohistiocytosis: an update for nephrologists. Int Urol Nephrol 2016;48:1291-1304.

2 Henter JI, Horne A, Arico M, et al: HLH-2004: diagnostic and therapeutic guidelines for hemophagocytic lymphohistiocytosis. Pediatr Blood Cancer 2007;48:124-131. 


\section{Case Reports in Oncology}

Diaz et al.: Minimal Change Disease as Initial Presentation of ALK-Positive Anaplastic Large-Cell Lymphoma in a Pediatric Patient

- Lehmberg K, Sprekels B, Nichols KE, Woessmann W, Muller I, Suttorp M, et al: Malignancy-associated haemophagocytic lymphohistiocytosis in children and adolescents. Br J Haematol 2015;170:539-549.

4 Pasqualini C, Minard-Colin V, Saada V, Lamant L, Delsol G, Patte C, et al: Clinical analysis and prognostic significance of haemophagocytic lymphohistiocytosis-associated anaplastic large cell lymphoma in children. Br J Haematol 2014;165:117-125.

5 Thaunat O, Delahousse M, Fakhouri F, Martinez F, Stephan JL, Noel LH, et al: Nephrotic syndrome associated with hemophagocytic syndrome. Kidney Int 2006;69:1892-1898. Da'as N, Polliack A, Cohen Y, Amir G, Darmon D, Kleinman Y, Goldfarb AW, Ben-Yehuda D: Kidney involvement and renal manifestations in non-Hodgkin's lymphoma and lymphocytic leukemia: a retrospective study in 700 patients. Eur J Haematol 2001;67:158-164.

-7 Kofman T, Zhang S, Copie-Bergman C, Moktefi A, Raimbourg Q, Francois H, et al: Minimal change nephrotic syndrome associated with non-Hodgkin lymphoid disorders: a retrospective study of 18 cases. Medicine (Baltimore) 2014;93:350-358.

-8 Rajasekaran S, Kruse K, Kovey K, Davis AT, Hassan NE, Ndika AN, et al: Therapeutic role of anakinra, an interleukin-1 receptor antagonist, in the management of secondary hemophagocytic lymphohistiocytosis/sepsis/multiple organ dysfunction/macrophage activating syndrome in critically ill children. Pediatr Crit Care Med 2014;15:401-408. 


\section{Case Reports in Oncology}

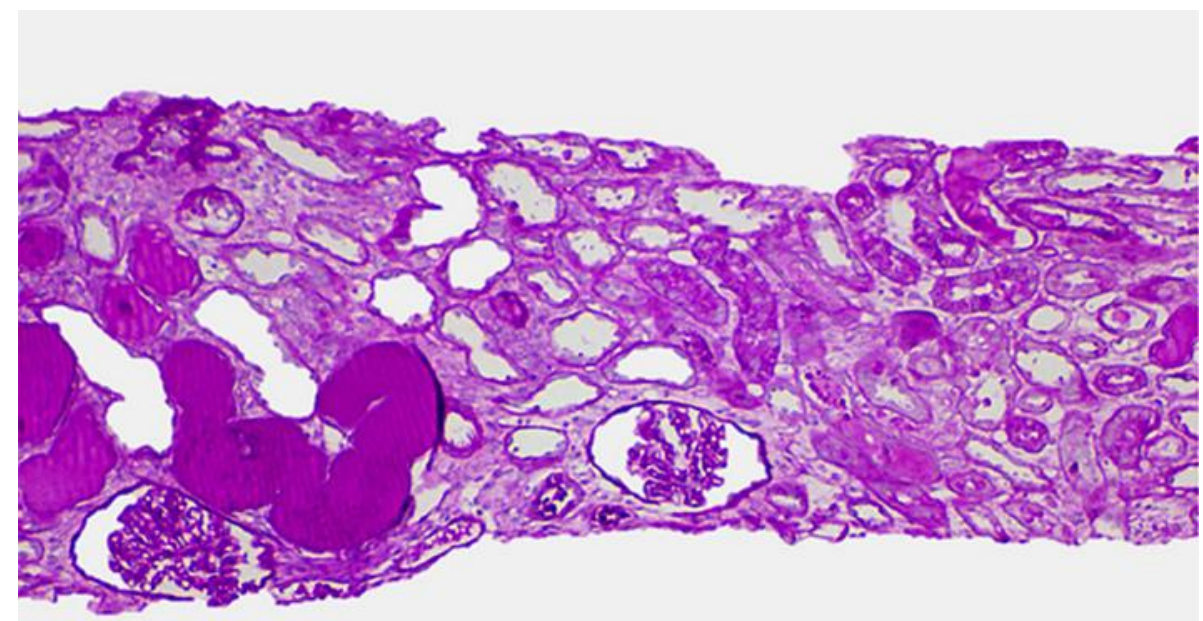

a

Diaz et al.: Minimal Change Disease as Initial Presentation of ALK-Positive Anaplastic Large-Cell Lymphoma in a Pediatric Patient

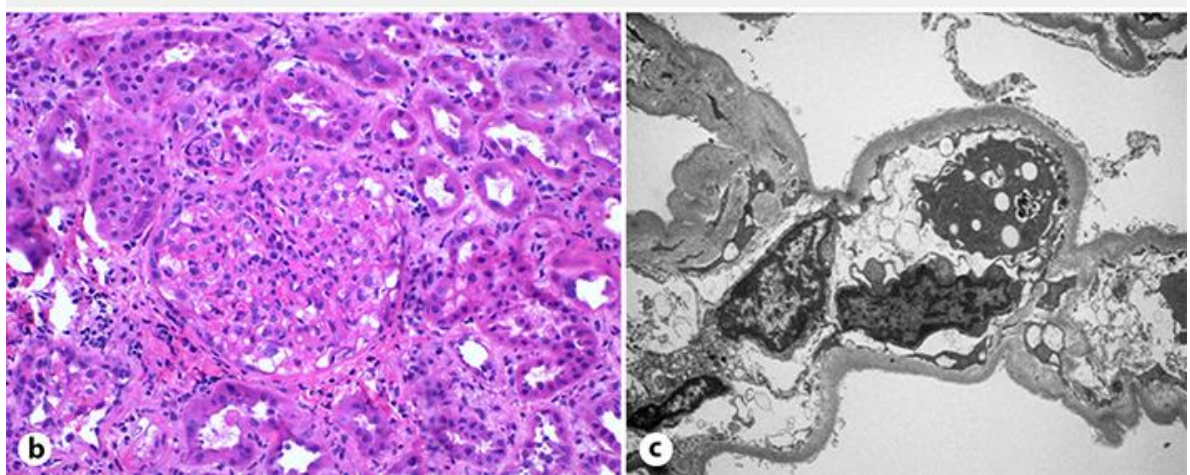

Fig. 1. Renal biopsy results. a Specimen shows minimal change glomerulonephritis and acute tubuleinterstitial nephritis. Glomeruli are intact but show reactive and edematous changes of the parietal and visceral epithelial cells. There is significant interstitial edema, acute tubular injury with associated mild interstitial nephritis, and exaggerated "minimal change" nephrosis. b The second renal biopsy shows progression (worsening) of the interstitial inflammation, edema, and glomerular epithelial changes. c Electron microscope shows podocyte effacement with no electron-dense deposits. 


\section{Case Reports in Oncology}
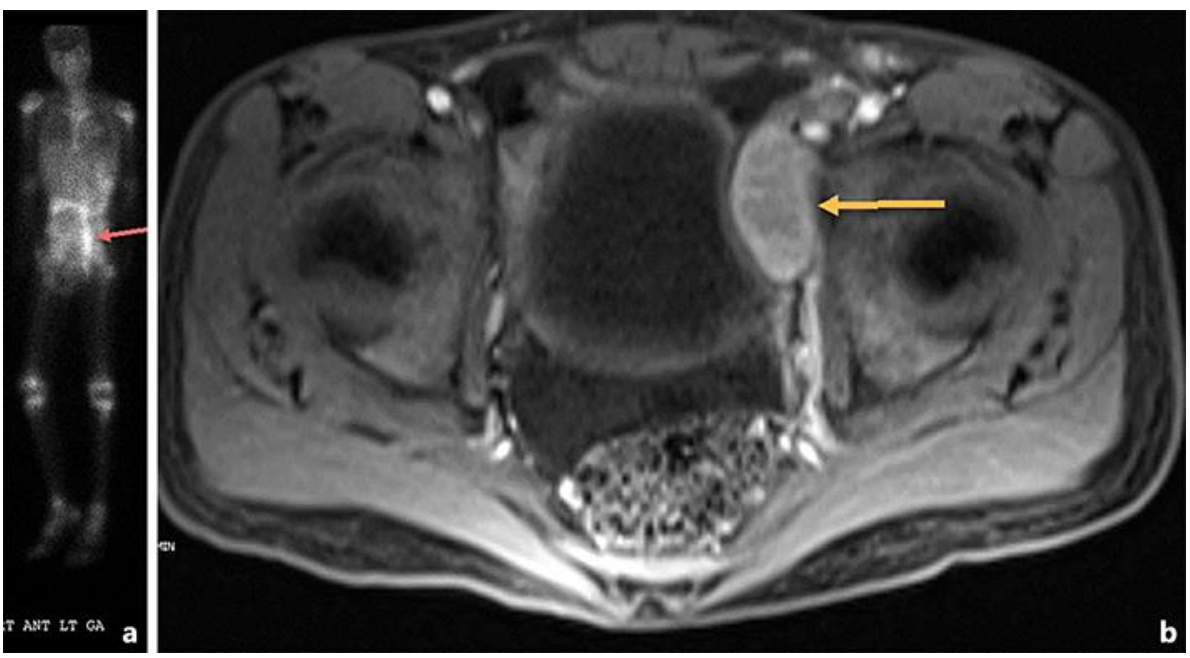

Fig. 2. Imaging studies showing marked lymphadenopathy. a Gallium scan shows intense gallium uptake along the left iliacus muscle extending linearly back up along the psoas and through the retroperitoneum (red arrow). b MRI of the pelvis shows a large, heterogeneously enhancing lymph node conglomerate extending from the left para-aortic region through the iliac chain into the left inguinal region. The left external iliac lymph node measures $3.8 \times 2.2 \mathrm{~cm}$ in maximum anterior-posterior and transverse dimensions impressing upon the left lateral bladder wall (orange arrow). 
Table 1. Clinical and laboratory findings

\begin{tabular}{|c|c|c|c|}
\hline & $\begin{array}{l}\text { At initial } \\
\text { presentation }\end{array}$ & $\begin{array}{l}\text { At HLH } \\
\text { diagnosis }\end{array}$ & $\begin{array}{l}\text { At ALCL } \\
\text { diagnosis }\end{array}$ \\
\hline Fever & present & present & present \\
\hline Splenomegaly & present & present & present \\
\hline Hemoglobin $<9$ g/dL & 11 & 8.1 & 7.7 \\
\hline Platelet count $<100 \times 10^{9} / \mathrm{L}$ & 148 & 107 & 76 \\
\hline Neutrophil count $<1.0 \times 10^{9} / \mathrm{L}$ & 3.23 & 0.61 & 1.66 \\
\hline Triglyceride $>54 \mathrm{mg} / \mathrm{dL}$ & 1,100 & 814 & 825 \\
\hline Fibrinogen $<150 \mathrm{mg} / \mathrm{dL}$ & 299 & 346 & 469 \\
\hline Natural killer cell activity & inadequate specimen & $\mathrm{N} / \mathrm{A}$ & $\mathrm{N} / \mathrm{A}$ \\
\hline Ferritin $>500 \mathrm{ng} / \mathrm{mL}$ & $\mathrm{N} / \mathrm{A}$ & 14,742 & 1,888 \\
\hline Soluble CD25 (soluble IL2R) $>2,400 \mathrm{U} / \mathrm{mL}$ & $\mathrm{N} / \mathrm{A}$ & 4,151 & $\mathrm{~N} / \mathrm{A}$ \\
\hline $\mathrm{LDH}, \mathrm{U} / \mathrm{L}$ & 2,117 & - & 280 \\
\hline Serum albumin, g/dL & 1.3 & 1.7 & 1.7 \\
\hline BUN, mg/dL & 71 & 39 & 19 \\
\hline Serum creatinine, $\mathrm{mg} / \mathrm{dL}$ & 8.02 & 5.14 & 1.31 \\
\hline $\mathrm{C} 3, \mathrm{mg} / \mathrm{dL}$ & 134 & - & - \\
\hline $\mathrm{C} 4, \mathrm{mg} / \mathrm{dL}$ & 82 & - & - \\
\hline Total cholesterol, mg/dL & 236 & - & 282 \\
\hline Urine protein & $300+$ & $300+$ & $300+$ \\
\hline AST, U/L & 168 & 103 & 25 \\
\hline ALT, U/L & 23 & 8 & 6 \\
\hline Total bilirubin, mg/dL & 0.1 & 0.2 & 0.1 \\
\hline INR & 1.3 & 1.2 & 1.1 \\
\hline Creatine kinase, U/L & 1,814 & 700 & 63 \\
\hline
\end{tabular}

N/A, not applicable or available; HLH, hemophagocytic lymphohistiocytosis; ALCL, anaplastic large-cell lymphoma; LDH, lactate dehydrogenase; BUN, blood urea nitrogen; AST, aspartate transaminase; ALT, alanine transaminase; INR, international normalized ratio. 
Table 2. Timeline of major events

\begin{tabular}{|c|c|c|c|}
\hline Event & $\begin{array}{l}\text { Hospital } \\
\text { day }\end{array}$ & Evaluation/workup & Description \\
\hline 1 & 0 & $\begin{array}{l}\text { Transferred to our hospital due to } \\
\text { concern for autoimmune disease }\end{array}$ & $\mathrm{N} / \mathrm{A}$ \\
\hline 2 & 5 & Rheumatologic workup & Negative \\
\hline 3 & 8 & Liver, muscle, and renal biopsies & $\begin{array}{l}\text { Nonspecific changes in liver and muscle } \\
\text { biopsies; minimal change disease on renal } \\
\text { biopsy (see Fig. 1) }\end{array}$ \\
\hline 4 & 9 & $\begin{array}{l}\text { Bone marrow biopsy } \\
\text { Lumbar puncture }\end{array}$ & Cytogenetics 46,XY \\
\hline 5 & 10 & Met 7 of 8 criteria for HLH diagnosis & $\begin{array}{l}\text { HLH thought to be secondary to viral } \\
\text { infection (rhinovirus and enterovirus) } \\
\text { Therapy with steroids (day 10-28) }\end{array}$ \\
\hline 6 & 24 & Recurrence of fevers & Negative infectious workup \\
\hline 7 & 25 & HLH genetic testing & Negative \\
\hline 8 & 26 & Removal of hemodialysis catheter & Completed 7 sessions of dialysis \\
\hline 9 & 32 & CT of the abdomen/pelvis & $\begin{array}{l}\text { Read as negative for lymphadenopathy or } \\
\text { abscess }\end{array}$ \\
\hline 10 & 33 & Administration of anakinra & $\begin{array}{l}\text { Discontinued } 1 \text { week later due to no } \\
\text { improvement and worsening fevers }\end{array}$ \\
\hline 11 & 40 & Nuclear medicine: gallium scan & see Figure 2a \\
\hline 12 & 41 & MRI of the abdomen/pelvis & see Figure $2 b$ \\
\hline 13 & 46 & Femoral lymph node biopsy & $\begin{array}{l}\text { ALK-positive anaplastic large-cell } \\
\text { lymphoma diagnosis }\end{array}$ \\
\hline 14 & 47 & Chemotherapy initiation & $\begin{array}{l}\text { Cyclophosphamide, intrathecal cytarabine, } \\
\text { vincristine, adriamycin, prednisone }\end{array}$ \\
\hline 15 & 72 & Discharged to home & $\mathrm{N} / \mathrm{A}$ \\
\hline 16 & 90 & Nephrotic syndrome resolution & Last albumin infusion \\
\hline 17 & 360 & $\begin{array}{l}\text { Relapse of ALK-positive anaplastic } \\
\text { large-cell lymphoma }\end{array}$ & Being evaluated for bone marrow transplant \\
\hline
\end{tabular}

N/A, not applicable; HLH, hemophagocytic lymphohistiocytosis. 